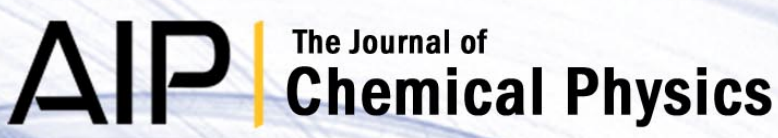

\section{Sculpting nanometer-sized light landscape with plasmonic nanocolumns}

Renaud Marty, Arnaud Arbouet, Christian Girard, Jérémie Margueritat, José Gonzalo et al.

Citation: J. Chem. Phys. 131, 224707 (2009); doi: 10.1063/1.3271794

View online: http://dx.doi.org/10.1063/1.3271794

View Table of Contents: http://jcp.aip.org/resource/1/JCPSA6/v131/i22

Published by the American Institute of Physics.

\section{Additional information on J. Chem. Phys.}

Journal Homepage: http://jcp.aip.org/

Journal Information: http://jcp.aip.org/about/about_the_journal

Top downloads: http://jcp.aip.org/features/most_downloaded

Information for Authors: http://jcp.aip.org/authors

\section{ADVERTISEMENT}
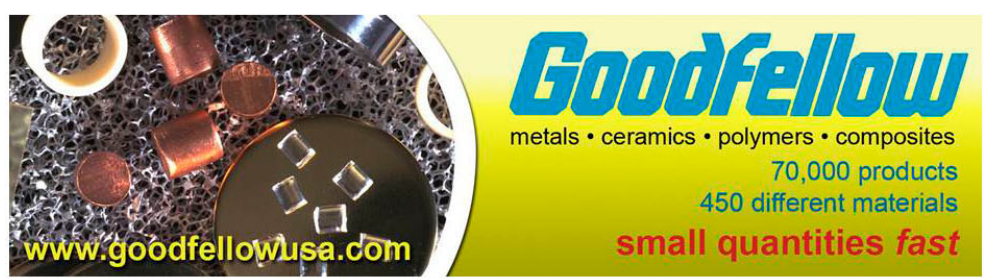


\title{
Sculpting nanometer-sized light landscape with plasmonic nanocolumns
}

\author{
Renaud Marty, ${ }^{1}$ Arnaud Arbouet, ${ }^{1}$ Christian Girard, ${ }^{1, a)}$ Jérémie Margueritat, ${ }^{1,2, b)}$ \\ José Gonzalo, ${ }^{2}$ and Carmen N. Afonso ${ }^{2}$ \\ ${ }^{1}$ CEMES, CNRS, Université Paul Sabatier, 29 rue Jeanne Marvig, Toulouse 31055, France \\ ${ }^{2}$ Laser Processing Group, Instituto de Optica, CSIC, Serrano 121, Madrid 28006, Spain
}

(Received 20 July 2009; accepted 15 November 2009; published online 11 December 2009)

\begin{abstract}
Plasmonic structures are commonly used to both confine and enhance surface electromagnetic fields. In the past ten years, their peculiar optical properties have given rise to many promising applications ranging from high density data storage to surface optical trapping. In this context, we investigated both far-field and near-field optical response of a collection of densely packed silver nanocolumns embedded in amorphous aluminum oxide using the discrete dipole approximation. In the far field, a good fit of the calculated to the experimental absorption spectra can only be achieved when in addition to interaction between neighboring nanocolumns, a nanorod shape with periodic shrinks mimicking the experimental morphology of the nanocolumns is used. In the near field, modulated field intensities following the nanocolumns distribution and tunable with the incident wavelength are predicted outside the region occupied by the nanocolumns. This plasmonic image transfer has a resolution of approximately $1.8 D$ where $D$ is the diameter of the nanocolumns that in our case is 2.4 nm. (C) 2009 American Institute of Physics. [doi:10.1063/1.3271794]
\end{abstract}

\section{INTRODUCTION}

The optical response of small metallic aggregates has extensively been investigated theoretically during the last 50 years. In the case of noble metal colloidal particles or lithographically designed structures, characterized by a permittivity $\epsilon_{m}(\omega)$, embedded in transparent materials of dielectric constant $\epsilon_{\text {env }}$, the interface between the two media introduces surface plasmon resonances (SPRs). ${ }^{1}$ In this context, a large amount of both theoretical and experimental investigations addressing either the linear or nonlinear optical properties has been performed on systems of increasing complexity. ${ }^{2-8}$ These studies evidenced the influence of size, shape, and environment on the properties of isolated nanoparticles. The potential applications of these metallic systems extend from colloidal markers, nanobiosensors, light emission enhancement devices, plasmonic circuitry, ${ }^{9,10}$ plasmonic tweezers, ${ }^{11}$ to stimulated emission control. ${ }^{12}$

Among all these objects, elongated particles (prolate spheroids, nanorods, nanowires, or nanoantennas) have been investigated in order to obtain new plasmonic functionalities. ${ }^{13-15}$ Generally, these objects exhibit two main optical resonances. ${ }^{16}$ The first one is related to the isotropic SPR band occurring in spherical particles that survives as a transverse SPR (TSPR). The second is associated with conduction electron oscillations along the major axis of the particle, the so-called longitudinal SPR (LSPR). As expected, the spectral position of the LSPR dramatically depends on the particle aspect ratio. ${ }^{17-19}$

With the improvement of current fabrication techniques, nanostructures involving a large number of entities such as

\footnotetext{
${ }^{\text {a)} E l e c t r o n i c ~ m a i l: ~ g i r a r d @ c e m e s . f r . ~}$

${ }^{b)}$ Present address: Denis Diderot University-Paris 7, Laboratoire ITODYS UMR 7086, 15 rue Jean de Baif, 75205 Paris Cedex 13, France.
}

self-assembled plasmonic nanostructures based on colloidal metal nanoparticles or arrays of metal nanoparticles have been fabricated. Besides the physical properties inherited from their individual building blocks, these new structures show original optical or acoustic properties due to interparticle coupling. ${ }^{20-22}$ For instance, interparticle electromagnetic coupling between metallic nanoparticles of a chain opens up the possibility of guiding light at the nanoscale. ${ }^{23}$ In this case, such collective effects are applied to develop new functionalities. They provide additional degrees of freedom to tailor the optical response of a complex nanostructure. ${ }^{24,25}$ Another example is the calculation of the near-field modulation produced by an array of $20 \mathrm{~nm}$ diameter oriented silver nanorods that has been proposed for subwavelength optical imaging with a resolution of $40 \mathrm{~nm}$ given by the rod size and spacing. ${ }^{26}$

Recently, self-assembled silver nanocolumns ( $\mathrm{NCls})$ have been fabricated using pulsed laser deposition. ${ }^{27}$ These nanostructures are composed of small silver NCls (diameter $2<D<3 \mathrm{~nm}$ and height $6<H<15 \mathrm{~nm}$ ) aligned perpendicularly to the substrate with an interparticle spacing comparable to the diameter of the $\mathrm{NCls}$, thus having much smaller features than the ones used for earlier calculations. ${ }^{26}$ The absorption cross section of these structures exhibits two bands with a smaller spectral splitting than expected in isolated silver $\mathrm{NCls}$ due to interparticle electromagnetic coupling. ${ }^{28}$ In addition, in the particular case of metallic nanoparticles with sizes in the sub-10-nm range, confinement effects will lead to a significant modification of the dielectric constant of the metal with respect to its bulk value and should be taken into account. ${ }^{29-31}$

The aim of this paper is to calculate the far-field and near-field effects produced by the densely packed array of silver NCls whose production and structural properties were reported elsewhere. ${ }^{27}$ The model, based on the discrete di- 

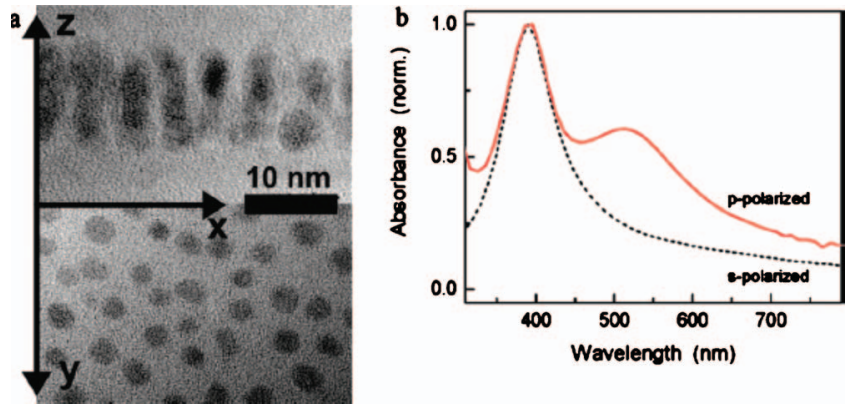

FIG. 1. (a) plan view and cross-section transmission electron microscopy image of the sample taken from Ref. 36. (b) Extinction spectra recorded at $75^{\circ}$ angle of incidence with p- (continuous line) and s- (dashed line) polarization.

pole approximation (DDA), ${ }^{32-34}$ is first used to fit the experimentally measured far-field response. The role of the dimension of the array and the detailed shape of the NCls (perfect or shrinked rods, with or without dispersion) is first studied separately. Appropriate selection of these parameters together with a fine adjustment of NCls separation lead finally to a very good fit of the experimental data. The near-field patterns at different distances of the array are finally calculated for different wavelengths and polarizations. The results show the possibility of sculpting nanometer-sized light landscape with silver NCls with sub-10-nm resolution. Although the direct observation of such extremely confined optical fields is difficult to achieve with standard scanning near-field optical microscopes (SNOMs), they could be imaged by applying new methods inherited from electron energy loss spectroscopy (EELS). ${ }^{8,35}$ Indeed, SNOM imaging of optical near fields around nanometer sized metallic particles with sufficient lateral resolution needs nanometric optical probes that are not yet available.

\section{SELF-ASSEMBLED SILVER NANOCOLUMNS}

Self-assembled silver NCls embedded in an amorphous $\mathrm{a}-\mathrm{Al}_{2} \mathrm{O}_{3}$ matrix have been produced by alternate pulsed laser deposition in vacuum $\left(10^{-6}\right.$ mbar $)$ using an ArF excimer laser (pulse duration of $20 \mathrm{~ns}$ full width half maximum, energy density of $1.9 \mathrm{~J} \mathrm{~cm}^{-2}$ ). The beam was sequentially focused on the surface of high-purity $\mathrm{Al}_{2} \mathrm{O}_{3}$ and $\mathrm{Ag}$ rotating targets. First, a layer of a- $\mathrm{Al}_{2} \mathrm{O}_{3}$ was deposited to act as substrate where a layer of $\mathrm{Ag}$ nanoparticles is formed following the Vollmer-Weber growth mode of metal on an oxide surface. Then the space among the Ag nanoparticles was filled almost completely with $\mathrm{Al}_{2} \mathrm{O}_{3}$, the uncovered part of the metal nanoparticles acting as a template during next deposition of Ag. The samples consisted in several cycles of ablation metal matrix, thus leading to a single layer of self-aligned NCls. Further details of the synthesis procedure as well as the morphological study of the samples using high resolution transmission electron microscopy (HRTEM) have been presented elsewhere. ${ }^{36}$ The sample selected for the present study contains NCls with a diameter of $2.4 \mathrm{~nm}$ and a height of $8.6 \mathrm{~nm}$ as it can be seen in the plan- and cross-section views included in Fig. 1 taken from Ref. 36. Unless otherwise stated, all calculations reported in this work are performed using this diameter and height. Figure 1 also shows the extinction spectra obtained at an angle of incidence of $75^{\circ}$ using either s- or p-polarized incident light. Taking into account the refractive index of the matrix, the propagation direction inside the material with respect to the axis of the NCls is $35^{\circ}$. In the case of s-polarized light a single band around $390 \mathrm{~nm}$ is observed which corresponds to the TSPR. In the case of p-polarized light two distinct resonances can instead be observed at 390 and $540 \mathrm{~nm}$, which correspond, respectively, to transverse and longitudinal SPRs as expected for elongated objects. However, the experimental spectral splitting between these resonances is clearly smaller than the one predicted by the Gans theory for isolated spheroidal particles of comparable aspect ratio or by numerical calculations of the optical properties of a single nanorod. ${ }^{37,38}$ This discrepancy suggests that electromagnetic coupling between the individual nanoobjects is playing a role. Indeed, Zong et al. ${ }^{39}$ investigated arrays of silver nanowires with much higher aspect ratio than our investigated sample but a comparable volume fraction of metal. They pointed out that interparticle electromagnetic interaction cannot be neglected in these systems. Jain and El-Sayed et al. ${ }^{40}$ demonstrated that the proximity between two adjacent nanorods leads to a modification of the absorption of the ensemble that strongly depends on the number of interacting nanoobjects and on their arrangement, but the dimensions and separation of the nanoobjects were close to one order of magnitude higher than in our case. In our system, the shorter interparticle separation and their larger number density can be responsible for even stronger modifications of the optical response with respect to isolated NCls. To address this point, we performed in the next section numerical calculations of the far-field optical response of dense arrays of self-assembled silver NCls.

\section{FAR-FIELD OPTICAL RESPONSE OF DENSE ARRAYS OF METALLIC NANOPARTICLES}

In a first step, to gain insight into the optical properties of these systems, we focused on the far-field optical properties of ideal arrays of silver NCls and computed their extinction, scattering, and absorption cross sections. In the following, we will only discuss the extinction spectra as absorption and scattering spectra will obviously display the same trend. The influence of the number of interacting nanorods, their shape, and dispersion have selectively been addressed.

\section{A. Volume discretization and cell polarizability}

We used the DDA which has now become a routine method to compute extinction, scattering, and absorption of light by nanostructures with arbitrary shapes for which no analytical solution of Maxwell's equation can be found. The DDA was developed by Purcell and Pennypacker ${ }^{41}$ and has been refined by different groups, in particular by Draine and co-workers. ${ }^{32,42-45}$ Unless otherwise stated, the following calculations have been done with the publicly available code DDSCAT 6.1. ${ }^{46}$ In the framework of the DDA, the target is approximated by a finite array of polarizable points placed in a cubic lattice with a spacing very small compared to the effective wavelength in the material. 
Every point is given an effective polarizability $\alpha_{i}(\omega)$ deduced from the incident wave vector $k$, the permittivity of the surrounding medium $\left(\mathrm{a}-\mathrm{Al}_{2} \mathrm{O}_{3}\right) \epsilon_{\text {env }}$, and the permittivity of silver at the frequency of the incident light $\epsilon_{m}(\omega)$ following the lattice dispersion relation prescription. ${ }^{47}$ All our calculations have been done assuming that the dielectric constant of the environment of the metallic nanoparticles is $\epsilon_{\mathrm{env}}=2.79$. Due to the very small size of the investigated metallic NCls, the modification of the dielectric constant of the metal due to the confinement has to be taken into account. ${ }^{1,30}$

\section{B. Confinement and related dissipation effects}

In bulk noble metals, the conduction band electrons have a quasi-free-electron behavior characterized by the following Drude-like contribution: ${ }^{48}$

$$
\epsilon^{(f)}(\omega)=1-\frac{\omega_{P}^{2}}{\omega\left(\omega+\imath \gamma_{o}(\omega)\right)},
$$

where $\omega_{P}$ is the plasma frequency, and $\gamma_{o}(\omega)$ is the electronic relaxation rate. The contribution of the other electrons to the dielectric constant leads to an additional contribution $\delta \epsilon^{(b)}(\omega) .{ }^{49}$ It is generally accepted that the dielectric constant in small metallic nanoparticles can be obtained from the bulk optical constants through the following modification of the relaxation rate of the Drude-like contribution:

$$
\gamma(\omega)=\gamma_{o}^{\prime}(\omega)+g_{s}(\omega) \frac{v_{F}}{R} .
$$

The first term $\gamma_{o}^{\prime}(\omega)$ is similar to the intrinsic relaxation rate of the bulk whereas the second is a consequence of the confinement of the electronic motion. Classically, this term can be understood as a limitation of the mean free path of the electrons due to scattering on the surface of the nanoparticle. A rigorous calculation of the transitions between the confined electronic eigenstates of a nanosphere yields a similar expression with a value of $g_{s}(\omega)$ very close to $1 .^{29-31}$

Due to the very small size of the investigated nanoparticles, the additional term will be very important. Indeed, for $R=3 \mathrm{~nm}, \hbar v_{F} / R \approx 300 \mathrm{meV}$, whereas in bulk silver, the intrinsic relaxation rate is 15 times smaller $\left(\hbar \gamma_{o} \approx 20 \mathrm{meV}\right){ }^{48}$ In the following calculations, we used the data from Palik ${ }^{50}$ modified as explained above with $g_{s}=1$.

\section{Self-consistency}

Following external optical excitation, every point of the lattice acquires a fluctuating dipole moment proportional to the local electric field. Both incident field and fields produced by the other dipoles will contribute to the local electric field. These mutual interactions can be expressed by the following self-consistent equation:

$$
\mathbf{E}\left(\mathbf{r}_{\mathbf{i}}, \omega\right)=\mathbf{E}_{\mathbf{0}}\left(\mathbf{r}_{\mathbf{i}}, \omega\right)+\sum_{j} \mathbf{T}\left(\mathbf{R}_{\mathbf{i j}}, \omega\right) \cdot \alpha_{j}(\omega) \cdot \mathbf{E}\left(\mathbf{r}_{\mathbf{j}}, \omega\right),
$$

where $\mathbf{T}\left(\mathbf{R}_{\mathbf{i j}}, \omega\right)$ is the field susceptibility in the surrounding medium that can be analytically written as

$$
\begin{aligned}
\mathbf{T}\left(\mathbf{R}_{\mathbf{i j}}, \omega\right)= & \frac{e^{i k R_{i j}}}{\epsilon_{\mathrm{env}}}\left[\frac{k^{2}}{R_{i j}^{3}}\left(\mathbf{R}_{\mathbf{i j}} \mathbf{R}_{\mathbf{i j}}-R_{i j}^{2} \mathbf{I}\right)+\left(\frac{1}{R_{i j}^{5}}-\frac{i k}{R_{i j}^{4}}\right)\right. \\
& \left.\times\left(3 \mathbf{R}_{\mathrm{ij}} \mathbf{R}_{\mathbf{i j}}-R_{i j}^{2} \mathbf{I}\right)\right],
\end{aligned}
$$

where $\mathbf{I}$ is the identity dyadic tensor, $\mathbf{R}_{\mathbf{i j}}=\mathbf{r}_{\mathbf{i}}-\mathbf{r}_{\mathbf{j}}$, and $k$ $=(\omega / c) \sqrt{\epsilon_{\text {env }}}$. The different dipoles will interact with the other dipoles via their electric fields. The $N$ equations above can be written as a matrix equation,

$$
\sum_{j=1}^{N} \mathbf{A}_{\mathbf{i}, \mathbf{j}}(\omega) \cdot \mathbf{E}\left(\mathbf{r}_{\mathbf{j}}, \omega\right)=\mathbf{E}_{\mathbf{0}}\left(\mathbf{r}_{\mathbf{i}}, \omega\right),
$$

with

$$
\mathbf{A}_{\mathbf{i}, \mathbf{j}}(\omega)=\mathbf{I} \delta_{i, j}-\alpha_{i}(\omega) \mathbf{T}\left(\mathbf{R}_{\mathbf{i j}}, \omega\right) .
$$

The self-consistent solution of this equation provides the dipole moments at every location of the lattice. From these dipole moments, absorption and scattering cross sections can be computed.

\section{Extinction cross section of a two-dimensional array of perfect silver nanorods}

In this subsection, we investigate the influence of the number of interacting silver nanorods of diameter $D$ on the far-field optical response of an ideal array. The nanorods are arranged side by side with their long axes perpendicular to the plane of the array. We investigate the cases of an incident plane wave polarized perpendicularly or parallel to the plane of the sample. The nanoparticles were organized on a square lattice with a regular spacing $d=2 D$. Figure 2 shows the extinction cross section for an incident electric field respectively parallel (a) or perpendicular to the long axis of the nanorods (b). The resonance apparent in Fig. 2(a) is due to the LSPR of the nanorods whereas the one evidenced in Fig. 2(b) originates from the TSPR. As shown in Fig. 1, in s-polarization only the TSPR is detected whereas both resonances are excited in p-polarization. As expected transverse and longitudinal modes show completely different behaviors as seen in Fig. 2. While the spectra of Fig. 2(a) reveal a significant blueshift of the LSPR as the number of nanorods increases, spectra in Fig. 2(b) indicate that the net shift of the TSPR is rather weak. ${ }^{51}$ The blueshift in Fig. 2(a) is a pure near-field effect since the near-field generated by a nanorod along the long axis of its neighbor nanorods has opposite direction than the incident field and thus will add constructively to its depolarization field. As a consequence, the restoring force acting on the electronic motion will increase and the LSPR will be shifted toward shorter wavelength. Figure 2(c) explicitly shows the evolution of the position of the longitudinal and TSPRs with the number of nanorods in the array. Applying periodic boundary conditions with DDSCAT 7.0, 52,53 the extinction cross section of an infinite array could be computed. The dashed (resp. dotted) line indicate the position of its longitudinal (resp. transverse) SPR. As can be seen in Fig. 2(c), the longitudinal mode unambiguously shifts from $906 \mathrm{~nm}$ in the case of a single nanorod to $596 \mathrm{~nm}$ for an infinite array. 

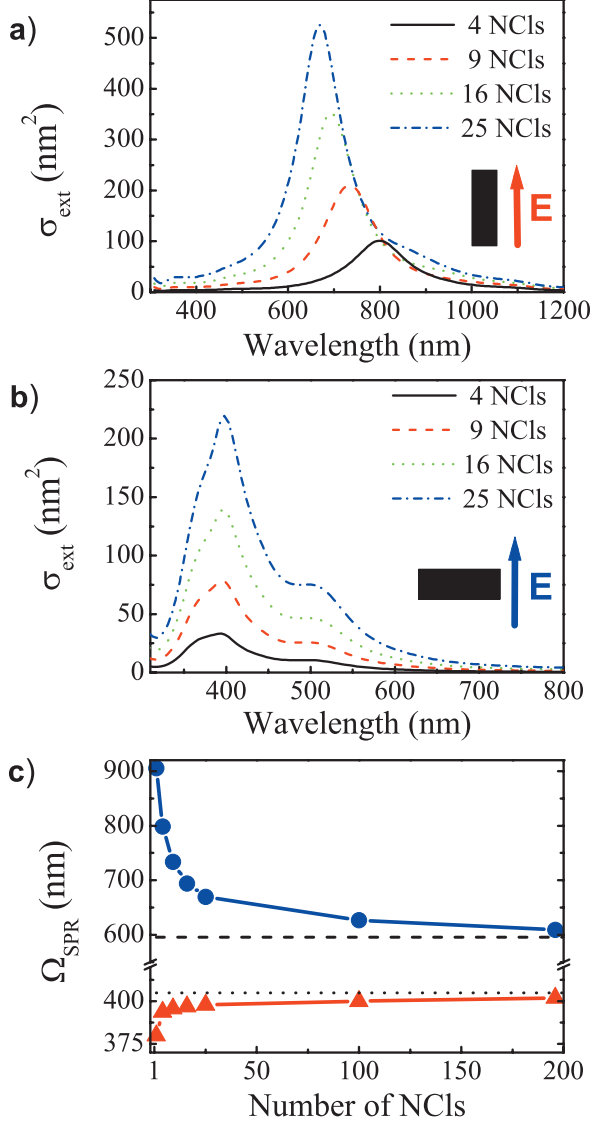

FIG. 2. Extinction cross section of a square array of silver nanorods for an incident electric field polarized along the long axis (a) or the short axis (b) of the nanorods. (c) Evolution of the position of the longitudinal and TSPRs with the number of nanorods in the array. The dashed (resp. dotted) line indicates the wavelength of longitudinal (resp. transverse) SPR of an infinite square array of nanorods.

However, at this point, the agreement between our calculations and the experimental results is not completely satisfactory and additional effects such as shape effects must be considered to explain the observed data.

\section{E. Shape column effect}

The experimentally produced metallic $\mathrm{NCls}$ have a shape that departs from the ideal rod shape used in the calculations and have in addition important shape dispersions as seen in Fig. 1(a). To evaluate the influence of both shape and shape dispersion we fixed the number of interacting nanorods to nine and compared results produced by the three different shapes schematically shown on top of Fig. 3: (i) identical nanorods, (ii) identical nanorods with periodic shrinks mimicking the real morphology, and (iii) "realistic" nanorods having shapes similar to case (ii) but with some shape dispersions. The aspect ratio, volume, and environment are the same in all three cases. As expected, Fig. 3 shows that shape has a dramatic impact on the optical response of the nanorods; perfect nanorods (i) have a slightly redshifted transverse plasmon resonance and their longitudinal plasmon resonance is more than three times more intense than for realistic nanorods (iii). Moreover, a shoulder clearly appears around $500 \mathrm{~nm}$ in case (i), while it is almost negligible in cases (ii) and (iii). It is also clear that cases (ii) and
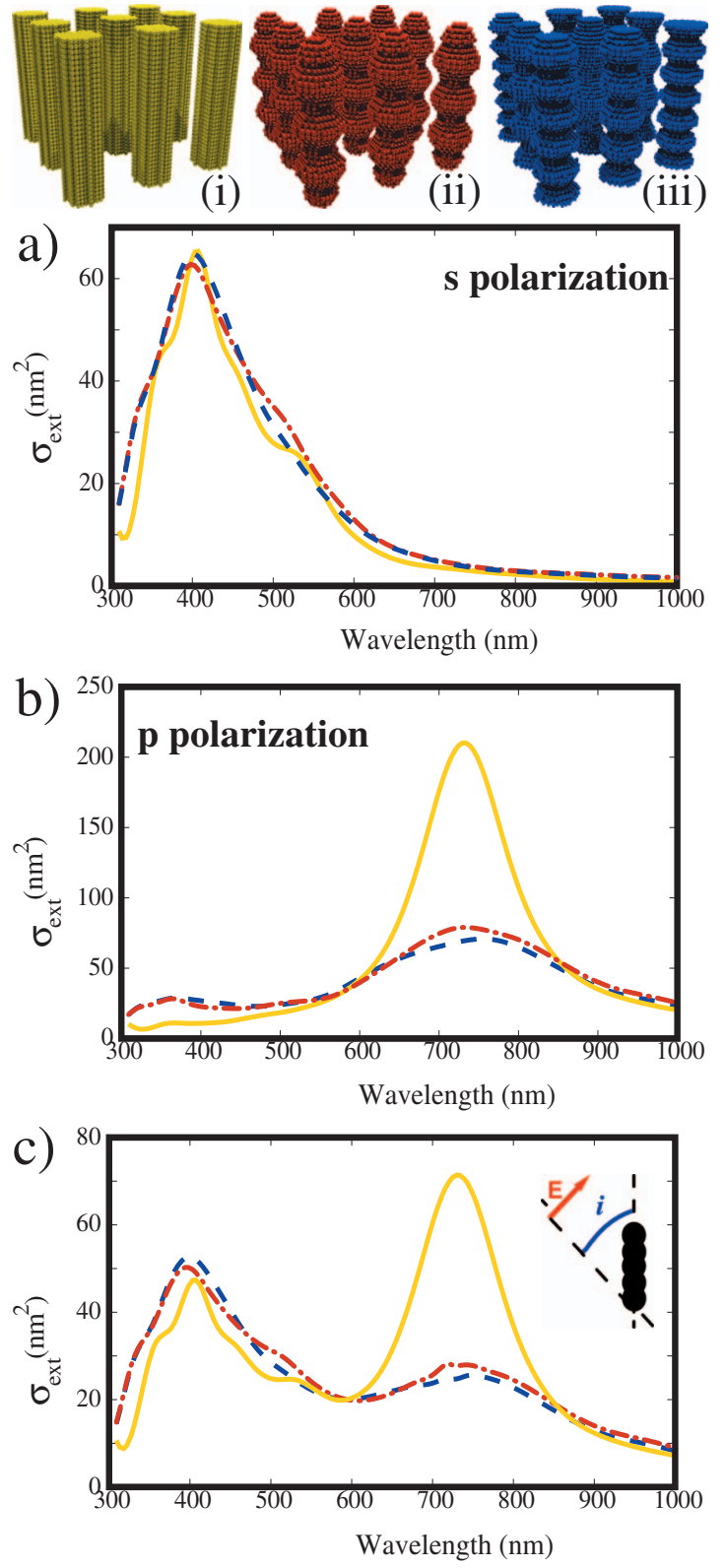

FIG. 3. Extinction cross section of an array of nine silver nanorods with (i) identical perfect rod shape (solid line), (ii) identical rod shape with periodic shrinks mimicking the real morphology of NCls (dash dotted), and (iii) shape similar to case (ii) but with shape fluctuations (dashed). (a) s-polarization. (b) p-polarization. (c) p-polarized electric field with incidence angle $i=35^{\circ}$.

(iii) give very similar results; small shape fluctuations among the shrinked nanorods have no major effect on the optical response of the ensemble. Comparison of Fig. 3(c) with Fig. 1(b) unambiguously shows that the perfect rod shape cannot account for the lower experimental extinction cross section at the LSPR.

In view of these results, we extended our calculations to a square array of 21 silver shrinked nanorods, adjusted the lattice spacing to $1.8 \mathrm{D}$ to fit the average interparticle separation of the real $\mathrm{NCls}$ and used p-polarized electric field and $35^{\circ}$ incident angle as in Fig. 1(b). After normalization at the TSPR, both experimental and calculated spectra are shown in Fig. 4 where it is seen that the agreement is excellent. It can thus be concluded that the correct value of the spectral split- 


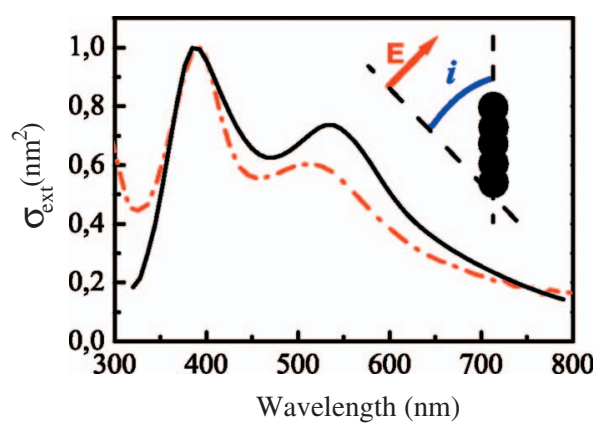

FIG. 4. Calculated extinction cross section of a square array of 21 silver shrinked nanorods (solid line) for a p-polarized electric field with incidence angle $i=35^{\circ}$ and corresponding experimental absorption spectrum (dash dotted).

ting between transverse and LSPRs in real NCls embedded in $\mathrm{Al}_{2} \mathrm{O}_{3}$ host can satisfactorily be reproduced by taking into account dissipation confinement correction, shape effects, and a large enough number of nanorods into the simulations.

Additional effects such as the nonlocality of the electronic response are expected to play a role in sub-10-nm noble metal nanoparticles. The impact of the spatial dispersion of the dielectric constant has been theoretically investigated in the case of spherical nanoparticles, ${ }^{55}$ nanowires, ${ }^{56}$ dimers, ${ }^{57}$ or two-dimensional arrays of metallic nanoparticles $^{58}$ and it has been shown that nonlocal effects can induce spectral shifts of the SPRs as well as a modification of their intensity. In fact, the issue of taking into account nonlocal effects in our calculations still remains both a theoretical and numerical challenge. For example, although a recent work by McMahon et al. ${ }^{54}$ reports significant advances to include these corrections, the numerical applications are still restricted to single two-dimensional plasmonics systems (infinite metallic nanowires of circular and triangular sections). This work indicates that nonlocal effects may be important in structures displaying apex features. In other cases, including rounded surface particles, these effects give rise to corrections to the local model. In our case, where the sample is composed of a distribution of rounded NCls, this correction has been neglected. In the future, we intend to include these phenomena by generalizing our fieldsusceptibility formalism. Finally, neglecting nonlocal effect does not modify the near field behaviors predicted in the next section (Sec. IV) (dark and bright contrasts) because the reduction in the coupling, appearing due to nonlocal effects in metal, does not affect the contrast mechanism that is mainly produced by the individual nanorods.

This first series of far-field simulations enabled us to validate a set of reliable dielectric data to describe the optical response of nanometric silver aggregates. Consequently, it can be used to predict near-field topography and intensity near these systems.

\section{NEAR-FIELD OPTICAL RESPONSE}

Within the framework of the volume description used in this paper, the electric field outside the objects, at the location $\mathbf{R}$, is merely deduced from the self-consistent polariza-

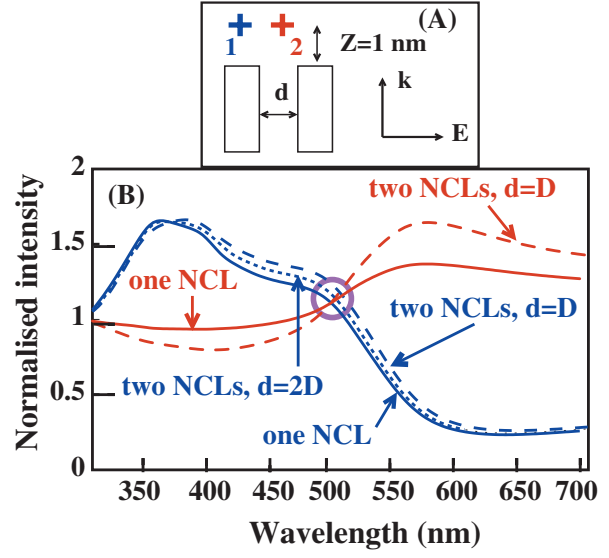

FIG. 5. (a) Investigated configuration: the incident electric field is perpendicular to the major axis of the nanorods. (b) Optical near-field spectra computed $1 \mathrm{~nm}$ above a single (solid line) or two silver nanorods separated by $2.4 \mathrm{~nm}$ (dashed) or $3.6 \mathrm{~nm}$ (dotted). The spectra have been calculated at location 1 (blue) or 2 (red). The metallic nano-objects are embedded in a- $\mathrm{Al}_{2} \mathrm{O}_{3}$.

tion $\mathcal{P}(\mathbf{r}, \omega)$ inside the metallic particles, provided that we know the field propagator $\mathbf{T}\left(\mathbf{r}, \mathbf{r}^{\prime}, \omega\right)$ of the bare sample [see Eq. (4)]. One can write

$$
\mathbf{E}(\mathbf{R}, \omega)=\mathbf{E}_{0}(\mathbf{R}, \omega)+\int_{\mathrm{vol}} \mathbf{T}(\mathbf{R}, \mathbf{r}, \omega) \cdot \mathcal{P}(\mathbf{r}, \omega) d \mathbf{r},
$$

where the integral runs over the volume occupied by the nanorods. This solution can be used to compute the normalized optical near-field intensity in the vicinity of the illuminated sample versus the position $\mathbf{R}$ :

$$
I(\mathbf{R})=|\mathbf{E}(\mathbf{R}, \omega)|^{2} /\left|\mathbf{E}_{o}(\mathbf{R}, \omega)\right|^{2} .
$$

As in the previous sections, the investigated metallic nanostructures are discretized in lattices of a total of $N$ points located at positions $\mathbf{r}_{i}$. The self-consistent Eq. (3) yields a linear system of $3 N$ equations. By its numerical inversion, the electric field is self-consistently calculated at every position $\mathbf{r}_{i}$ inside the nanostructure. In a second step, the electric field at any location $\mathbf{R}$ is calculated by applying the propagation Eq. (7). Due to the very high dielectric constant of silver, these calculations required a fine discretization of the nanostructure. The distance between adjacent points must be fixed at about $0.3 \mathrm{~nm}$. In order to get more insight on the impact of the interparticle coupling, we calculated using this formalism, the near-field intensity distribution of the electric field intensity produced by different arrays of silver nanorods. The numerical results are presented in Figs. 5-7 where $Z$ is the distance between the top end of the nanorods and the observation plane as indicated in Fig. 5(a). As expected intuitively, the excitation wavelength $\lambda$ is still an important parameter in the very near field. Generally, the excitation of surface plasmons causes a characteristic field enhancement compared to the incident electromagnetic wave. Two different sets of data can be used to characterize the spectroscopic properties in the very near field:

(i) The near-field optical spectrum $I\left(\mathbf{R}=\mathbf{R}_{0}, \lambda\right)$ that will give the variation of the normalized near-field optical 


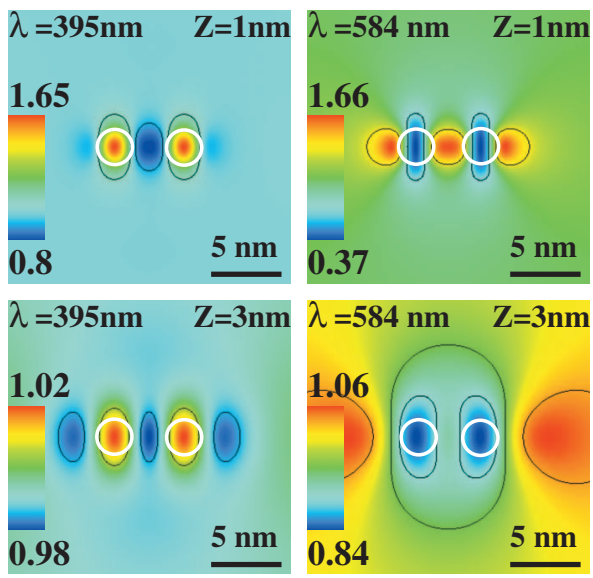

FIG. 6. Normalized optical near-field intensity distribution in a plane at height $Z$ above two silver nanorods embedded in a- $\mathrm{Al}_{2} \mathrm{O}_{3}$. The incident electric field is in the same configuration as Fig. 5(a) with wavelength $\lambda$. The nanorods represented by white circles are $2.4 \mathrm{~nm}$ in diameter and separated by $2.4 \mathrm{~nm}$.

intensity recorded at a given location $\mathbf{R}_{0}$ above the sample versus the incident wavelength $\lambda$.

(ii) Two-dimensional near-field optical intensity maps $I\left(X, Y, Z=Z_{0}, \quad \lambda=c s t\right)$ that provide an image of the optical near field for a selected wavelength.

To illustrate the original near-field optical properties that can be expected around silver nanorods, we present in Fig. 5 five typical spectra computed at two different locations labeled (1) (blue cross) and (2) (red cross) on the side view. The more striking feature is the presence around the wavelength $\lambda_{c}=500 \mathrm{~nm}$ of a crossing point [see the magenta circle in Fig. 5(b), where the different spectral lines cross). This wavelength $\lambda_{c}$ splits the optical range into two domains in which more or less bright or dark contrast can be expected. These spectra show that it is possible to produce either highly localized light depressions or enhancements near silver NCls by controlling the excitation of the localized plasmons. In addition, mutual interactions between these metallic nanorods tend to reinforce the amplitude between dark and bright contrasts [see dashed curves in Fig. 5(b)].

This is first confirmed on near-field optical intensity maps presented in Fig. 6 where two heights $Z$ and two incident wavelengths have been considered for the case of two silver nanorods. Figure 7 illustrates this on larger near-field maps performed above a seven silver nanorod array. The analysis of these results raises the following comments.

(i) Although the system is illuminated in the s-polarized mode, each silver $\mathrm{NCl}$ can display a positive contrast in the electric near-field intensity maps, in particular when $\lambda<\lambda_{c}$, and give rise to large field gradients in the proximity of the top end of the $\mathrm{NCl}$. Within this frequency range $\left(\lambda<\lambda_{c}\right)$ unlike what happens with dielectric nanodots or $\mathrm{NCls}$ for which a dark contrast is expected and was measured, ${ }^{59,60}$ the occurrence of bright contrasts with s-polarized light is directly related to the singularity of the real part of the polarizability appearing when tuning the incident wavelength near a plasmon resonance. The physics of this pecu-
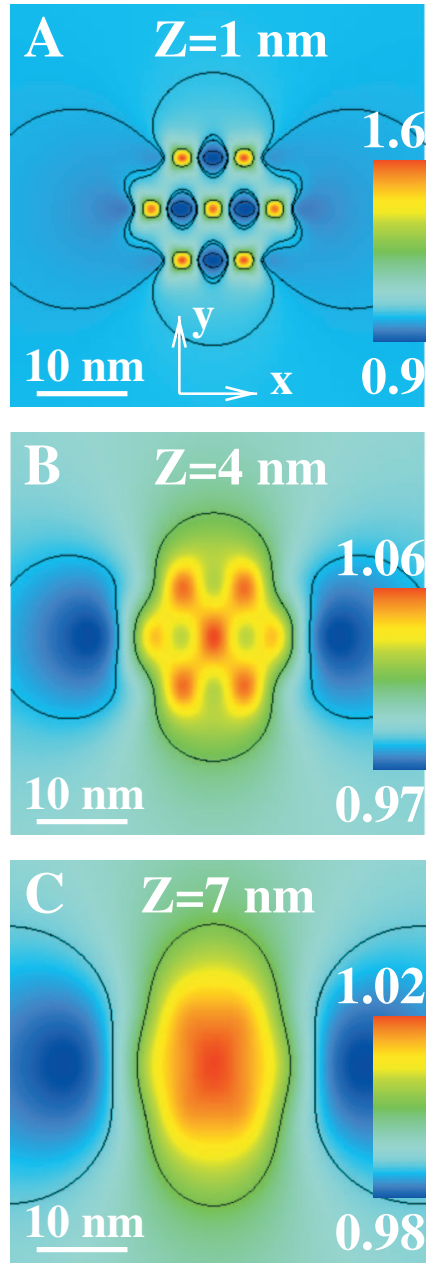

FIG. 7. Normalized optical near-field intensity distribution computed at three different heights $Z$ above seven silver nanorods $2.4 \mathrm{~nm}$ in diameter. (a), (b), and (c) represent the evolution of the intensity map when increasing the observation plane distance $Z$. Both spacing $d$ between nanorods and incident wavelength $\lambda$ are fixed at 2.4 and $390 \mathrm{~nm}$, respectively.

liar optical phenomenon can be expressed by a simple analytical relation that defines the contrast above a single $\mathrm{NCl}$ of global polarizability $\mathbf{A}(\omega)$ :

$\eta_{s}(\omega)=\frac{|\mathbf{E}(\mathbf{R}, \omega)|^{2}}{\left|\mathbf{E}_{0}(\mathbf{R}, \omega)\right|^{2}}-1$.

This coefficient is negative (resp. positive) for a dark (resp. bright) contrast. When the observation point $\mathbf{R}$ is located just on the top of the particle, i.e., when $\mathbf{R}=(0,0, R)$, the sign and the magnitude of this coefficient provide direct information on the light confinement phenomenon. In the case of metallic materials, $\eta_{s}$ can be merely obtained from a previous result [cf. Eq. (20) of Ref. 60],

$\eta_{s}(\omega)=-\frac{2 \Re\left(A_{\perp}(\omega)\right)}{R^{3}}$,

where $A_{\perp}(\omega)$ represents the transverse polarizability component of the $\mathrm{NCl}$. When scanning the incident wavelength from blue to red colors, the real part of $A_{\perp}(\omega)$ exhibits two negative and positive ranges of values and vanishes for $\lambda=\lambda_{c}$. That explains the exis- 
(A)
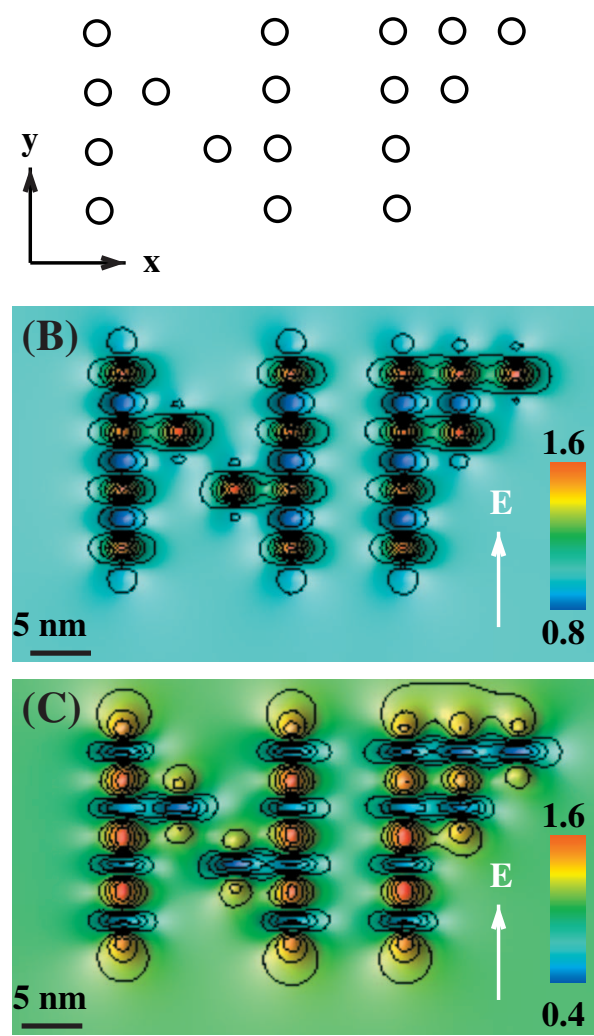

FIG. 8. Normalized optical near-field intensity distribution computed for two different wavelengths above silver nanorods, $2.4 \mathrm{~nm}$ in diameter, and organized in the plane (XOY) as the acronym $\mathbf{N ~ F}$ (for near field). The observation plane is located $1 \mathrm{~nm}$ from the $\mathrm{NCl}$ tops. (a) Top view of the pattern. [(b) and (c)] Near-field intensity maps computed for $\lambda=400 \mathrm{~nm}$ and $\lambda=600 \mathrm{~nm}$, respectively.

tence of the crossing point in the near-field zone as well as the division of the optical spectrum into zones with dark and bright contrasts. Recently, a similar behavior has been predicted by Ono et al. ${ }^{26}$ in which it is shown from finite difference time domain simulations that silver nanorod arrays are equivalent to subwavelength optical imaging systems.

(ii) According to Eq. (10), the dielectric behavior observed in Ref. 59 is recovered when $\lambda>\lambda_{c}$. This result is consistent with an off-resonant regime for which we have a large detuning between incident wavelength and transverse plasmon resonance wavelength.

(iii) The near-field optical confinement region is highly sensitive to the $Z$ distance (cf. Figs. 6 and 7). Indeed, in contrast with the case of larger microfabricated metallic structures, ${ }^{10,61-63}$ beyond a distance range $Z$ $>4 \mathrm{~nm}$, the optical near field spreads out over several nanorod sites without significant spatial modulation. We also checked that, beyond about $7 \mathrm{~nm}$, all subwavelength-sized features completely vanish and are progressively reduced to a single light spot.

The original near-field optical effects investigated in Figs. 5-7 are summarized in Fig. 8, where the near-field intensity above an array of 17 vertical silver $\mathrm{NCls}$ arranged to form the pattern $\mathbf{N} \mathbf{F}$ is shown. As illustrated in Fig. 8(a), the scanned area is centered around this pattern and the system is illuminated in normal incidence. In addition to a contrast reversal phenomenon, similar to that described in Fig. 5, and which occurs when passing from $\lambda=400 \mathrm{~nm}$ (TSPR) to $\lambda=600 \mathrm{~nm}$, the two images reproduce the $\mathbf{N} \mathbf{F}$ pattern with both excellent contrast and lateral resolution. This field expulsion outside the region occupied by the NCls can be remotely controlled by wavelength but also by tuning the incident polarization, so that possible applications in the local trapping of cold atoms as well as in the subwavelength optical image control might be realized with this kind of plasmonic systems. Because of the usual finite tip-size effects, direct imaging of such extremely confined optical fields will remain challenging in the future with standard SNOM instrumentation. However, other nanotechnology investigation methods could be applied. Recently, Colliex and co-workers ${ }^{8}$ demonstrated impressive plasmon mappings over single metallic nanocrystals by scanning a monochromatic focused electron beam. This well-established method, based on the EELS signal detection, allows subnanometric resolutions to be reached and could be the adequate experimental instrument to measure the near-field optical distribution and associated wavelength dependent behaviors predicted in this section.

\section{CONCLUSION}

The results of calculations based on volume discretized method (DDA and Green dyadic method) demonstrated the importance of near-field electromagnetic interparticle coupling in the optical response of a collection of self-assembled silver nanorods or NCls. In particular, for interparticle distances comparable to the nanorods $/ \mathrm{NCls}$ diameter, the farfield extinction spectra are dramatically modified by the near-field optical interaction between the nanoobjects.

The near-field map simulations clearly demonstrate that samples containing oriented elongated nano-objects provide a unique means for nanometer-sized patterning of initially flat optical near fields. The optical patterning is optimized when working at incident wavelengths close to collective plasmon modes of the array.

Finally, the results presented in this paper not only illustrate the complex properties of electromagnetic near fields in the vicinity of self-assembled $\mathrm{NCl}$ arrays but also help to identify new efficient subwavelength properties by analyzing their near-field optical images versus the distance between arrays and observation areas.

\section{ACKNOWLEDGMENTS}

This work was supported by the computing facility center CALMIP of the University Paul Sabatier of Toulouse (France) and by Grant No. MAT2005-6508-c02-01, Ministerio de Educacion y Ciencia (Spain).

\footnotetext{
${ }^{1}$ U. Kreibig and M. Vollmer, Optical Properties of Metal Clusters (Springer, Berlin, 1995).

${ }^{2}$ C. Flytzanis, F. Hache, M. C. Klein, D. Ricard, and P. Roussignol, Progress in Optics (North Holland, Amsterdam, 1991), Vol. XXIX.
} 
${ }^{3}$ J. P. Kottmann, O. J. F. Martin, D. R. Smith, and S. Schultz, Phys. Rev. B 64, 235402 (2001).

${ }^{4}$ M. C. Daniel and D. Astruc, Chem. Rev. (Washington, D.C.) 104, 293 (2004).

${ }^{5}$ C. Girard and E. Dujardin, J. Opt. A, Pure Appl. Opt. 8, S73 (2006).

${ }^{6}$ G. Lévêque and O. J. F. Martin, Opt. Lett. 31, 2750 (2006).

${ }^{7}$ H.-Y. She, L.-W. Li, S. J. Chua, W.-B. Ewe, O. J. F. Martin, and J. R. Mosig, J. Appl. Phys. 104, 064310 (2008).

${ }^{8}$ J. Nelayah, M. Kociak, O. Stephan, F. J. Garcia de Abajo, M. Tence, L. Henrard, D. Taverna, I. Pastoriza-Santos, L. M. Liz-Marzan, and C. Colliex, Nat. Phys. 3, 348 (2007).

${ }^{9}$ W. L. Barnes, A. Dereux, and T. W. Ebbesen, Nature (London) 424, 824 (2003)

${ }^{10}$ G. P. Wiederrecht, Eur. Phys. J.: Appl. Phys. 28, 3 (2004).

${ }^{11}$ M. Righini, G. Volpe, C. Girard, D. Petrov, and R. Quidant, Phys. Rev. Lett. 100, 186804 (2008).

${ }^{12}$ J. Seidel, S. Grafström, and L. Eng, Phys. Rev. Lett. 94, 177401 (2005).

${ }^{13}$ N. R. Jana, L. Gearheart, and C. J. Murphy, Adv. Mater. (Weinheim, Ger.) 13, 1389 (2001)

${ }^{14}$ P. Zijlstra, A. L. Tchebotareva, J. W. M. Chon, M. Gu, and M. Orrit, Nano Lett. 8, 3493 (2008).

${ }^{15}$ L. Douillard, F. Charra, Z. Korczak, R. Bachelot, S. Kostcheev, G. Lerondel, P.-M. Adam, and P. Royer, Nano Lett. 8, 935 (2008).

${ }^{16}$ K. L. Kelly, E. Coronado, L. L. Zhao, and G. C. Schatz, J. Phys. Chem. B 107, 668 (2003).

${ }^{17}$ S. Link and M. A. El-Sayed, J. Phys. Chem. B 103, 8410 (1999).

${ }^{18}$ M. B. Mohamed, V. Volkov, S. Link, and M. A. El-Sayed, Chem. Phys. Lett. 317, 517 (2000).

${ }^{19}$ E. Dujardin, L. B. Hsin, C. R. C. Wang, and S. Mann, Chem. Commun. 2001, 1264.

${ }^{20}$ R. Atkinson, W. R. Hendren, G. A. Wurtz, W. Dickson, A. V. Zayats, P. Evans, and R. J. Pollard, Phys. Rev. B 73, 235402 (2006).

${ }^{21}$ D. P. Lyvers, J.-M. Moon, A. V. Kildishev, V. M. Shalaev, and A. Wei, ACS Nano 2, 2569 (2008)

${ }^{22}$ A. Pinchuk and G. Schatz, Appl. Phys. B: Lasers Opt. 93, 31 (2008).

${ }^{23}$ S. A. Maier, P. G. Kik, and H. A. Atwater, Appl. Phys. Lett. 81, 1714 (2002).

${ }^{24}$ X. Wu, J. Zhang, and Q. Gong, Opt. Express 17, 2818 (2009).

${ }^{25}$ Z. Yang, W. H. Ni, X. S. Kou, S. Z. Zhang, Z. H. Sun, L. D. Sun, J. F. Wang, and C. H. Yan, J. Phys. Chem. C 112, 18895 (2008).

${ }^{26}$ A. Ono, J. Kato, and S. Kawata, Phys. Rev. Lett. 95, 267407 (2005).

${ }^{27}$ J. Margueritat, J. Gonzalo, C. N. Afonso, M. I. Ortiz, and C. Ballesteros, Appl. Phys. Lett. 88, 093107 (2006).

${ }^{28}$ J. Margueritat, J. Gonzalo, C. N. Afonso, A. Mlayah, D. B. Murray, and L. Saviot, Nano Lett. 6, 2037 (2006).

${ }^{29}$ A. Kawabata and R. Kubo, J. Phys. Soc. Jpn. 21, 1765 (1966).

${ }^{30}$ J. Perenboom, P. Wyder, and F. Meier, Phys. Rep. 78, 173 (1981).

${ }^{31}$ F. Hache, D. Ricard, and C. Flytzanis, J. Opt. Soc. Am. B 3, 1647 (1986)

${ }^{32}$ B. T. Draine, Astrophys. J. 333, 848 (1988).

${ }^{33}$ D. Barchiesi, C. Girard, O. J. F. Martin, D. Van Labeke, and D. Courjon, Phys. Rev. E 54, 4285 (1996).
${ }^{34}$ G. Colas des Francs, C. Girard, J. Weeber, C. Chicane, T. David, A. Dereux, and D. Peyrade, Phys. Rev. Lett. 86, 4950 (2001).

${ }^{35}$ F. J. García de Abajo and M. Kociak, Phys. Rev. Lett. 100, 106804 (2008).

${ }^{36}$ J. Burgin, P. Langot, A. Arbouet, J. Margueritat, J. Gonzalo, C. N. Afonso, F. Valle, A. Mlayah, M. D. Rossell, and G. Van Tendeloo, Nano Lett. 8, 1296 (2008).

${ }^{37}$ S. S. Chang, C. W. Shih, C. D. Chen, W. C. Lai, and C. R. C. Wang, Langmuir 15, 701 (1999).

${ }^{38}$ K. G. Thomas, S. Barazzouk, B. I. Ipe, S. T. S. Joseph, and P. V. Kamat, J. Phys. Chem. B 108, 13066 (2004).

${ }^{39}$ R.-L. Zong, J. Zhou, Q. Li, B. Du, B. Li, M. Fu, X.-W. Qi, L.-T. Li, and S. Buddhudu, J. Phys. Chem. B 108, 16713 (2004).

${ }^{40}$ P. K. Jain and M. A. El-Sayed, J. Phys. Chem. C 112, 4954 (2008).

${ }^{41}$ E. M. Purcell and C. R. Pennypacker, Astrophys. J. 186, 705 (1973).

${ }^{42}$ G. H. Goedecke and S. G. O'Brien, Appl. Opt. 27, 2431 (1988).

${ }^{43}$ W. T. Doyle, Phys. Rev. B 39, 9852 (1989).

${ }^{44}$ V. V. Varadan, A. Lakhtakia, and V. K. Varadan, IEEE Trans. Antennas Propag. 37, 800 (1989).

${ }^{45}$ J. J. Goodman, B. T. Draine, and P. J. Flatau, Opt. Lett. 16, 1198 (1991).

${ }^{46}$ B. T. Draine and P. J. Flatau, J. Opt. Soc. Am. A Opt. Image Sci. Vis. 11, 1491 (1994)

${ }^{47}$ B. T. Draine and J. Goodman, Astrophys. J. 405, 685 (1993).

${ }^{48}$ P. B. Johnson and R. W. Christy, Phys. Rev. B 6, 4370 (1972).

${ }^{49}$ H. Ehrenreich and H. R. Philipp, Phys. Rev. 128, 1622 (1962).

${ }^{50}$ E. D. Palik, Handbook of Optical Constants of Solids (Academic, San Diego, 1998)

${ }^{51}$ P. K. Jain, S. Eustis, and M. A. El-Sayed, J. Phys. Chem. B 110, 18243 (2006).

${ }^{52}$ B. T. Draine and P. J. Flatau, "User Guide to the Discrete Dipole Approximation Code DDSCAT 7.0," arXiv:0809.0337v5.

${ }^{53}$ B. T. Draine and P. J. Flatau, J. Opt. Soc. Am. A Opt. Image Sci. Vis. 25, 2593 (2008).

${ }^{54}$ J. M. McMahon, S. K. Gray, and G. C. Schatz, Phys. Rev. Lett. 103, 097403 (2009).

${ }^{55}$ R. Ruppin, Phys. Rev. B 45, 11209 (1992).

${ }^{56}$ M. Boustimi, J. Baudon, P. Fron, and J. Robert, Opt. Commun. 220, 377 (2003).

${ }^{57}$ F. J. Garcia de Abajo, J. Phys. Chem. C 112, 17983 (2008).

${ }^{58}$ V. Yannopapas, J. Phys. C 20, 325211 (2008).

${ }^{59}$ J. C. Weeber, E. Bourillot, A. Dereux, J. P. Goudonnet, Y. Chen, and C. Girard, Phys. Rev. Lett. 77, 5332 (1996).

${ }^{60}$ C. Girard, A. Dereux, and J. C. Weeber, Phys. Rev. E 58, 1081 (1998).

${ }^{61}$ J. R. Krenn, R. Wolf, A. Leitner, and F. R. Aussnegg, Opt. Commun. 137, 46 (1997).

${ }^{62}$ T. Klar, M. Perner, S. Grosse, G. von Plessen, W. Spirkl, and J. Feldmann, Phys. Rev. Lett. 80, 4249 (1998).

${ }^{63}$ M. Salerno, N. Félidj, J. R. Krenn, A. Leitner, F. R. Aussenegg, and J. C. Weeber, Phys. Rev. B 63, 165422 (2001). 